Regards sur l'économie allemande

Bulletin économique du CIRAC

$73 \mid 2005$

Varia

\title{
Le cas Deutsche Börse AG et ses conséquences pour la place financière allemande
}

Markus Gabel

\section{(2) OpenEdition}

\section{Journals}

Édition électronique

URL : http://journals.openedition.org/rea/214

DOI : $10.4000 /$ rea. 214

ISBN : 978-2-8218-0842-3

ISSN : 1965-0787

\section{Éditeur}

CIRAC

\section{Édition imprimée}

Date de publication : 1 octobre 2005

Pagination : 27-36

ISSN : 1156-8992

\section{Référence électronique}

Markus Gabel, «Le cas Deutsche Börse AG et ses conséquences pour la place financière allemande », Regards sur l'économie allemande [En ligne], 73 | octobre 2005, document 3, mis en ligne le 25 juin 2008, consulté le 20 avril 2019. URL : http://journals.openedition.org/rea/214 ; DOI : 10.4000/rea.214 


\section{Le cas Deutsche Börse AG et ses conséquences pour la place financière allemande}

\section{Markus Gabel}

Entre le 13 décembre 2004 et 10 mai 2005, le paysage financier allemand a changé de visage. En décembre 2004, Werner Seifert, président du directoire de Deutsche Börse AG, rend publique une offre d'achat hostile sur le London Stock Exchange. A peine cinq mois plus tard et après l'échec de l'OPA, il annonce le 9 mai 2005 sous la pression massive d'actionnaires, notamment de deux hedge fonds britanniques, sa démission immédiate. A la fin de l'année, il est suivi par Rolf Breuer, président du conseil de surveillance. Pour la première fois en Allemagne, des actionnaires ont pesé lourdement non seulement sur l'orientation stratégique d'une entreprise, mais également sur le choix de l'équipe dirigeante. La mutation de la Deutschland AG serait-elle plus avancée qu'il ne le semblait encore récemment? En tout cas, les conséquences de ces événements risquent d'être profondes, non seulement pour l'entreprise Deutsche Börse qui cherche une nouvelle stratégie, mais également pour la place financière de Francfort. Par ailleurs, la consolidation du paysage boursier européen, encore très morcelé, surtout par rapport aux marchés américains, reste plus que jamais à l'ordre du jour.

La concurrence boursière entre Londres et Francfort est ancienne. Durant les années 1990, l'enthousiasme était immense à Francfort quand les banques allemandes ont racheté quelques fleurons de la banque d'investissement britannique, donnant naissance à la Dresdner Bank Kleinwort Benson et à la Deutsche Bank Morgan Grenfell. La puissance de la Bundesbank était à son apogée, et la décision d'installer la nouvelle Banque centrale européenne donnait un atout indiscutable à Francfort. En 2005, l'enthousiasme est retombé. Comme centre bancaire, Francfort a subi une nette perte d'importance ces dernières années. Le nombre de banques étrangères qui y sont implantées baisse depuis 2003 ; il dépasse aujourd'hui tout juste le niveau de 1994. Mais c'est surtout la faible rentabilité des banques allemandes qui relègue la finance allemande au deuxième rang en Europe. Le récent rachat de HVB par Unicrédito témoigne de cette évolution. Certes, les banques allemandes ont dépassé la crise de 2001/02, et leur rentabilité s'est nettement améliorée, mais - à l'exception de Deutsche Bank - elles restent loin des stars anglo-saxonnes.

Dans ce contexte, la Deutsche Börse AG (DBAG) fait figure d'exception. Sous la direction de W. Seifert, elle est devenue l'opérateur de marché le plus rentable d'Europe. Avec son système de négociation Xetra, elle est aussi technologiquement en pointe, surtout par rapport à Londres qui enregistre un retard dans la modernisation des opérations. La capitalisation boursière illustre ce décalage : alors que la valeur des entreprises cotées à la bourse de Londres est supérieure de plus de $50 \%$ à celle de leurs homologues cotées à Francfort, la capitalisation boursière de la DBAG est de loin la plus élevée de tous les opérateurs de marché, dépassant celle du London Stock Exchange (LES) de plus de $300 \%$. Cette success story avait commencé dès la création de la Deutsche Börse AG en 1993, issue de la fusion entre la Frankfurter Wertpapierbörse (l'ancienne bourse des valeurs) et le Kassenverein (la caisse centrale de dépôt
La place financière de Francfort en perte de vitesse

Deutsche Börse AG : une succes story sans équivalent 
L'intégration verticale, une stratégie gagnante

Eurex : seul véritable succès au plan européen

La prise de Londres échoue pour la deuxième fois

La communauté financière de Francfort divisée sur le projet des titres) ; elle s'est poursuivie après son introduction en bourse en 2001. Ces transformations représentent une des innovations les plus importantes de la place financière de Francfort, entraînant un changement fondamental : celui de la mutation de la bourse de Francfort, une place régionale plutôt tranquille, en un opérateur technologique en quête de rentabilité et poursuivant une stratégie bien précise. Cette mutation est étroitement liée au nom du président $W$. Seifert et à sa double stratégie : faire croître l'entreprise (intégration verticale et croissance externe) et œuvrer à la consolidation du paysage boursier européen.

L'organisation d'une bourse est une activité qui n'offre qu'une marge d'expansion limitée : c'est essentiellement la rationalisation des opérations qui permet d'augmenter les bénéfices. Certes, grâce à son monopole, l'opérateur peut conserver une partie des gains et les distribuer plutôt aux actionnaires qu'en faire bénéficier les utilisateurs. Mais ce monopole n'est qu'une question de temps, car bientôt les utilisateurs primaires, les banques d'investissements et les particuliers, exigent eux aussi l'accès à cette rente. La DBAG a tenté de résoudre ce problème par son modèle d'intégration verticale: depuis le rachat de Clearstream en 2002, l'entreprise réunit les activités de négociation, compensation et paiement-règlement. L'évolution de la rentabilité atteste la supériorité de ce modèle par rapport aux modèles horizontaux des concurrents (en Europe, seule la bourse de Milan poursuit la même stratégie). A un moment où la LES et Euronext enregistrent des baisses de leur chiffre d'affaires, la DBAG bénéficie de la plus forte hausse de ses recettes (plus de $13 \%$ au second trimestre 2005, ce qui laisse présager un Ebitda de plus de 600 millions $€$ pour l'année 2005). L'entreprise porte ainsi tous les attributs d'une valeur de croissance.

Dans le champ de la consolidation boursière européenne, les succès de la DBAG sont plus modestes. Outre l'absorption de Clearstream, il s'agit notamment de la création d'Eurex (par fusion entre la Deutsche Terminbörse DTB et son équivalent suisse, Soffex) en 1997, qui est devenu rapidement le plus grand marché à terme du monde. Mais depuis, les échecs s'enchaînent : un premier projet de fusion avec la LSE (le «iX ») échoue en 2000 à cause de la résistance des banques et brokers britanniques ; la même année naît Euronext, fruit du regroupement de trois bourses continentales (Paris, Bruxelles et Amsterdam). En 2001, Euronext, devenu le principal concurrent en Europe continentale, réussit à racheter Liffe, le marché à terme londonien. Durant l'été 2004, la DBAG échoue dans sa tentative d'OPA sur la bourse suisse SWX. Encore plus récemment, Euronext et Borsa Italiana obtiennent la majorité dans MTS, la plus grande plateforme de négociation d'obligations. La création d'Eurex reste ainsi le seul véritable succès de la Deutsche Börse au plan européen. Quant à la filiale américaine créée début 2004, elle affronte la rude concurrence des marchés locaux, en particulier du Chicago Board of Trade. Une décision sur l'avenir de cet engagement est attendue pour cet automne.

Le 13 décembre 2004, la DBAG crée la surprise en annonçant une offre d'achat de la LES au prix de 530 pence l'action pour un volume total de près de 2 milliards $€$. Le moment est bien choisi, car Euronext ne dispose que de très peu de réserves liquides après avoir décidé en 2004 de verser un dividende exceptionnel à ses actionnaires. Or, contre toute attente, Euronext, qui risque la marginalisation, entre dans la bataille; mais sans annoncer un prix - seulement son intention. Le 6 mars, la DBAG retire son offre sous la pression de ses actionnaires, dont deux hedge fonds britanniques, $\mathrm{TCl}$ et Atticus, rejoints par des institutionnels comme Fidelety, Merill Lynch et Generali Asset management.

\section{L'échec de l'OPA de DBAG, conséquence d'une aliénation}

Ce retrait n'est finalement pas très surprenant. Contrairement à Paris où toute la communauté financière soutient l'offre, les milieux financiers allemands étaient dès le début divisés sur les perspectives de l'opération. D'un côté il y 
avait ceux qui, à l'instar de la bourgmestre de Francfort, considèrent que le projet témoignait de la puissance de l'entreprise et de la place financière de Francfort ; mais très rapidement, les sceptiques, dont la Bundesbank, se sont fait entendre. Pour eux, le risque était trop grand qu'un rachat du marché londonien entraîne à terme le transfert des principales activités de marché vers la capitale britannique. Non seulement la taille du marché londonien, mais aussi le fait que plus de $40 \%$ des activités sur Xetra proviennent déjà de Londres plaident en faveur d'une concentration sur la place londonienne. Par ailleurs, le souvenir de 2000 et du projet iX est encore trop vif. A l'époque, W. Seifert ne voulait laisser à Francfort que le Neuer Markt, celui des valeurs de croissance, mais ce dernier a fermé son activité en 2002 suite à l'effondrement des cours de près de $90 \%$. Werner Seifert a certes essayé de rassurer, promettant notamment que le siège de la future structure demeurerait à Francfort, mais il n'a pas pu offrir une vision convaincante pour son site. Cela étonne peu, car la hiérarchie des priorités ne faisait jamais de doute pour Seifert : avant tout le destin de l'entreprise, conformément à la loi sur les sociétés anonymes qui stipule que la direction n'est liée qu'à l'intérêt de l'entreprise.

L'erreur de la direction de la DBAG était double: d'abord, de croire que ses plus grands adversaires se situent de l'autre côté du Rhin et ainsi de focaliser sa stratégie exclusivement sur Londres ; ensuite, de supposer acquis l'aval des actionnaires pour l'opération de fusion - reflet parfait de 40 ans d'une Deutschland $A G$ où l'influence des actionnaires est traditionnellement restreinte du fait de celle des banques (via le système des voix mandatées: Depotstimmrecht, voir REA 45/00) et de la cogestion (voir REA 72/05). Le contrôle se fait plus en interne, surtout par le conseil de surveillance, et les actionnaires se contentent de valider les décisions du directoire (modèle stakeholder). Lors de la première cotation de la DBAG en 2001, la société était détenue majoritairement par des investisseurs allemands, notamment des banques. Mais ces derniers ont successivement vendu leurs participations, profitant de l'excellente performance du titre pour améliorer leur bilan. Durant l'offre d'achat, la rotation dans l'actionnariat s'est accélérée, de sorte que lors de la l'assemblée générale du 25 mai 2005, seuls $7 \%$ du capital étaient encore détenus par des actionnaires allemands. L'entreprise est désormais majoritairement contrôlée par des investisseurs anglo-saxons dont le plus important, $\mathrm{TCl}$, détient $8 \%$ du capital.

Deutsche Börse a perdu ses actionnaires d'origine (en \%)

\begin{tabular}{|ccc|}
\hline Actionnaires : & Fin 2004 & $\mathbf{1 0}$ mai $\mathbf{2 0 0 5}$ \\
allemands $^{\text {a) }}$ & & $\mathbf{7}$ \\
britanniques $^{\text {b) }}$ & 26 & $\mathbf{4 8}$ \\
américains $^{\text {b) }}$ & 35 & $\mathbf{2 9}$ \\
autres $^{\text {Cours de bourse (en } €)}$ & 24 & $\mathbf{1 6}$ \\
\hline
\end{tabular}

Source: Frankfurter Allgemeine Zeitung, 11-05-2005. a) Essentiellement des banques. b) Essentiellement des fonds. c) Le niveau le plus haut a été atteint le 12-09-2005 avec 77,41€, ce qui correspond à une hausse de $100 \%$ depuis l'introduction en février 2001 ; l'essentiel de cette performance a été atteint depuis août 2004 .

Cette internationalisation de l'actionnariat ne s'est pas traduite dans le management où dominent toujours les 'anciens', surtout dans le conseil de surveillance. Mais ce manque d'adaptation a eu également pour conséquence un processus d'aliénation du côté des anciens actionnaires, des utilisateurs, tout comme des autorités de tutelle et de 'leur' bourse, fournissant un terrain favorable à la stratégie choisie par R. Breuer et W. Seifert : attaquer les hedge fonds en leur reprochant de n'être que des spéculateurs. Mais cette vision populiste, reflétée par le récent débat pré-électoral sur la capitalisme (lors duquel on a assimilé les investisseurs internationaux et notamment les hedge fonds à des essaims de sauterelles annihilant tout sur leur passage), est peu convaincante. Les hedge fonds critiquent le prix d'achat considéré comme trop élevé et doutent de la
Une direction en déphasage avec ses propres actionnaires

L'absence d'unanimité sur le rôle des hedge fonds 
Un management mis 'sous tutelle' par ses actionnaires

La recherche d'une nouvelle stratégie : trois options profitabilité globale de l'opération. Mais leurs critiques vont plus loin : ils souhaitent notamment une restructuration du conseil de surveillance, afin qu'il soit plus en phase avec la structure des actionnaires. Comme alternative au projet de rachat, les détracteurs proposent une autre affectation des réserves liquides sous forme d'un dividende exceptionnel - une stratégie qui satisfait les intérêts de leurs donateurs de capitaux; dans le cas de $\mathrm{TCl}$, ce sont des universités, des fondations et des assurances. Certes, on peut s'interroger sur la pérennité de l'engagement des fonds, mais même s'ils se retirent à moyen terme, une telle vente n'a rien à voir avec la fermeture d'une usine, car d'autres investisseurs prennent le relais.

\section{Une entreprise rentable en quête d'une nouvelle stratégie}

Le résultat des votes lors de l'assemblée générale de la DBAG du 25 mai est sans précédent pour l'entreprise : sur la base d'une présence de $60 \%$ des voix (plus du double de 2004), aucune motion présentée par le directoire n'a obtenu de majorité. A ceci s'ajoute le refus du quittus du conseil de surveillance, fait quasi inédit en Allemagne. L'assemblée générale a ainsi largement suivi l'avis des opposants, votant l'augmentation du dividende et un large programme de rachat d'actions. Mais le plus contraignant pour l'avenir de la société est certainement le refus de donner au directoire le pouvoir d'augmenter le capital sans l'accord des actionnaires - pourtant essentiel pour réaliser rapidement une OPA. Combiné à l'augmentation du dividende, ce refus place la DBAG dans une situation délicate : à l'avenir, l'entreprise est de facto obligée de prendre l'accord des actionnaires avant chaque opération de croissance externe (financement de fusions ou acquisitions), car la voie d'un financement sans accord des actionnaires (emprunts ou obligations) modifierait trop fortement le rating de l'entreprise. Ce dernier risque d'ailleurs également d'être affecté par le programme de rachat d'actions, car il réduit la base de capitaux propres.

Dans cette situation, il n'est pas certain que la DBAG reste capable de jouer un rôle actif dans la consolidation boursière européenne. Trois options sont envisageables. La première est la poursuite, certes à vitesse moindre, de la stratégie actuelle, c'est-à-dire de la croissance organique par la conquête de nouveaux marchés et l'élargissement de la gamme de produits. L'option deux est le démembrement de la société. Les nouveaux actionnaires, notamment les fonds britanniques, pourraient exiger la vente de Clearstream qui est certes très rentable (marge opérationnelle de $35 \%$ en 2004), mais qui monopolise également beaucoup de capitaux propres, car l'activité d'une chambre de compensation nécessite un excellent rating. TCI pourrait ainsi poursuivre sa stratégie de dividende, car Clearstream est estimé à environ 2 milliards $€$. Pour la DBAG, une telle cession représenterait un choc sans précédant car elle couperait l'entreprise de ses racines historiques. L'autre cession possible, celle d'Eurex, serait moins douloureuse, mais sans son marché à terme, la DBAG ne pèse plus lourd et risque de devenir un enjeu des divers intérêts présents en Europe. La question du démembrement est également au centre de la troisième option, celle d'un rapprochement, voire d'une fusion, avec un opérateur de marché européen. Avec Euronext par exemple, la mauvaise relation personnelle entre les présidents n'y faisant plus obstacle depuis le départ de W. Seifert. Mais Euronext possède son propre marché à terme (Liffe) et son propre système de compensation (Euroclear) ; la Commission européenne pourrait donc refuser le rapprochement. Enfin, Euronext semble peu intéressée par cette option et se prépare au rachat de la LSE. L'autre candidat au rapprochement est la bourse suisse SWX, surtout depuis que la DBAG a proposé à son patron actuel, Reto Francioni, la présidence du directoire. Le conseil de surveillance de la DBAG doit confirmer cette nomination le 10 octobre prochain. Le nouveau président devra rapidement définir la nouvelle stratégie de la société. Car avec l'introduction prochaine en bourse des grands opérateurs nord-américains, de nouveaux 
concurrents apparaîtront sur le continent européen. Parallèlement, la place de la DBAG par rapport aux bourses régionales allemandes doit être redéfinie.

\section{DBAG et bourses allemandes régionales}

Le panorama boursier allemand reste marqué par la structure fédérale du pays. Malgré une tendance à la concentration des bourses régionales (fusion de Berlin et Brême en 2002), Francfort reste dans une position dominante incontestable, qu'il s'agisse du parquet (Parketthandel) ou du système automatisé Xetra. Ce dernier couvre entre $50 \%$ (valeurs étrangères) et $95 \%$ (valeurs du DAX) des transactions en Allemagne, le reste se partageant entre le parquet (5 à $45 \%$ ) et les bourses régionales (1 à $10 \%)$. Ces dernières sont au nombre de six : Berlin-Brême, Hambourg, Stuttgart, Hanovre, Düsseldorf et Munich.

Afin de freiner leur lente perte de vitesse, les bourses régionales poursuivent depuis plusieurs années une stratégie de spécialisation, notamment dans le segment des petites valeurs familiales et des valeurs de croissance. Leurs tentatives d'y acquérir une masse critique rencontrent un succès très limité. Hambourg compte à peine une dizaine d'entreprises dans son «Start-up Market », Brême et Düsseldorf ont déjà refermé leurs segments respectifs et Munich, après son échec avec le «Top Quality Segment », fermé en 2002, vient de lancer une nouvelle tentative avec «M-access », destiné aux entreprises du Mittelstand. L'autre forme de spécialisation concerne les utilisateurs. Toutes les bourses régionales tentent de séduire les investisseurs particuliers (par les horaires ou par la possibilité de passer également des ordres très petits) mais également avec un succès limité, car depuis 2002, Xetra accepte des ordres unitaires (de taille 1). Berlin et Stuttgart ont plus de succès. Ainsi, Berlin a pu conquérir une position respectable dans le domaine des valeurs étrangères, notamment par rapport à l'Europe de l'Est. Et Stuttgart est même une place incontestable dans le domaine des produits dérivés (son segment Euwax traite $85 \%$ des warrants cotés en Allemagne).

Tant que les bourses régionales se maintiennent en zone de bénéfice (c'est le cas actuellement) et continuent de profiter de la tutelle (et protection) politique régionale (via les autorités respectives du Land), leur disparition n'est pas à l'ordre du jour. Mais elles pourraient subir un changement fonctionnel. L'occasion pourrait être la cinquième loi de promotion financière $(5$. Finanzmarktförderungsgesetz) actuellement en préparation, dont l'un des objectifs est l'unification de la structure et de la régulation boursières. Ainsi, les bourses régionales pourraient devenir des subordonnées dans un système boursier centralisé (comme le sont les Landeszentralbanken vis-à-vis la Bundesbank) en devenant par exemple des agences de bourse accueillant et conseillant les entreprises régionales désireuses de se transformer en entreprise cotée. La place financière allemande y gagnerait en transparence pour l'investisseur étranger.

La Deutsche Börse AG est, avec la BCE, un atout indiscutable pour Francfort. Toutes deux remplissent d'importantes fonctions de service public. Mais depuis son introduction en bourse, la DBAG a abandonné le principe de couverture des coûts (Kostendeckung) de l'ancien opérateur boursier et recherche désormais la rentabilité. Le conflit entre missions de service public (l'organisation et l'accès aux marchés financiers et à l'information, la R\&D dans le domaine des technologies de marché) et shareholder value est ainsi inévitable. Ce conflit en cache un deuxième qui oppose les intérêts des propriétaires de l'opérateur de marché (recherchant des bénéfices élevés) et ceux des utilisateurs qui demandent des conditions d'accès peu onéreuses. Tous les grands opérateurs boursiers étant aujourd'hui cotés, le problème se pose partout. Certes, la cotation accentue la concurrence et oblige les opérateurs à améliorer leur technologie et à réduire leurs coûts, mais en même temps, elle comporte le risque d'une prise de contrôle par des acteurs étrangers, non-spécialistes ou non intéressés aux besoins des entreprises locales (par exemple les PME allemandes). L'autorité de tutelle (ministère de l'Economie du Land) et l'autorité de régulation (la Bundesanstalt für Finanzdienstleistungsaufsicht, BaFin) pourraient être incitées à renforcer leur tutelle et à pousser les banques à s'engager à nouveau plus activement auprès des opérateurs boursiers.

\section{Gouvernance d'entreprise et Deutschland AG : une mutation irréversible}

Malgré la gravité du conflit au sein de la DBAG, la direction - c'est-à-dire le directoire et le conseil de surveillance - a agi dans le cadre de la réglementation des sociétés anonymes. Certes, elle aurait pu demander l'avis des actionnaires quant à l'opération sur le LSE. Mais selon la loi sur les sociétés par

Missions publiques vs. shareholder value
La loi donne des pouvoirs quasi illimités au directoire 
La loi $A k t G$ pousse à une confrontation personnelle

Renforcement du rôle des actionnaires

Ouverture à l'international et rentabilité font bon ménage actions (Aktiengesetz, AktG), le directoire n'a besoin que du soutien du conseil de surveillance. Dans sa jurisprudence (" doctrine Holzmüller »), la Cour de cassation fédérale (Bundesgerichshof) a listé quelques exceptions à cette règle, mais elles ne concernent pas le cas d'une fusion-acquisition. La loi AktG donne une grande latitude d'action au directoire par rapport au conseil de surveillance et lui confère pleine autonomie par rapport aux actionnaires : l'intérêt de l'entreprise prime, il revient au directoire de le définir et d'agir en conséquence ; sauf, bien sûr, dans certains cas où le conseil de surveillance doit donner son aval (par exemple sur des sujets concernant les salariés). Mais ce dernier peut tout au plus empêcher une mesure, jamais il ne peut prendre l'initiative.

Pour l'investisseur étranger, surtout anglo-saxon, cette construction est étonnante, voire incompréhensible, car il est habitué à une consultation ex ante. Aux temps de la Deutschland AG, cette situation n'a jamais vraiment posé de problème, car l'actionnariat était concentré et clairement représenté dans le conseil de surveillance, de même que les représentants des salariés au titre de la cogestion (voir REA 72/05). La situation est tout autre dans le cas d'un actionnariat très dispersé. Or aux termes de la loi $A K t G$, un conflit sur la stratégie de la direction ou une mesure ne peut être désamorcée en dernier recours que par la voie des personnes. Ainsi, au lieu de peser directement sur les décisions, les actionnaires doivent faire le détour via le conseil de surveillance par la menace d'une recomposition du conseil lors d'une assemblée générale (souvent extraordinaire). C'est ainsi que le directoire peut ensuite être influencé et, cas extrême, renouvelé. Cela explique pourquoi le conflit dans la DBAG s'est transformé en un conflit de personnes : la direction n'ayant pas, ou trop tard, cherché le contact avec les actionnaires, ces derniers ont été obligés d'exercer leur pression sur le conseil de surveillance, essentiellement via les médias et par des contacts informels.

Le cas de la DBAG est sans précédent : pour la première fois, les actionnaires d'une grande entreprise rentable ont inversé la stratégie du directoire malgré le soutien que lui apportait le conseil de surveillance et poussé à la démission les deux principaux dirigeants. Pour éviter d'autres cas de ce type, le gouvernement peut être amené à légiférer, par exemple sur la base d'une « doctrineHolzmüller » élargie, c'est-à-dire d'une définition plus précise des cas dans lesquels la direction doit demander l'avis des actionnaires. Plus probable semble une refonte du Code de corporate governance qui inciterait les entreprises à s'engager dans la voie de la consultation des actionnaires, la définition des cas précis relevant des entreprises. Quoi qu'il en soit, la nouvelle situation incite le management à intensifier son dialogue avec les actionnaires et le grand public ; la transparence des entreprises en sortira renforcée. Plus généralement, le cas de la DBAG pose la question du contrôle du management. Selon la loi AktG, le lieu unique du contrôle est le conseil de surveillance. II n'y a pas de raison de changer ce principe, dès lors que le conseil reflète la structure des actionnaires. Certes, le mandat des membres du conseil s'étend généralement sur 5 ans une durée trop longue pour des cas extrêmes comme celui de la DBAG, où les changements dans la structure de l'actionnariat se sont faits en l'espace de quelques semaines. Mais en général, ce mandat leur permet de veiller à l'équilibre entre le court et le long terme et de jouer le rôle de contre-poids face au directoire. Les actionnaires, longtemps négligés outre-Rhin, sortiront gagnants de ces changements, même si parfois il faut les prendre un peu par la main, comme le fait la récente loi sur l'intégrité d'entreprise et la modernisation du droit de recours (Gesetz zur Unternehmensintegrität und Modernisierung des Anfechtungsrechts, Umag) qui vise, entre autres, à développer leur présence aux assemblées générales.

L'évolution de la structure des actionnaires de DBAG n'est pas un cas isolé. Depuis la défiscalisation de la vente de participations en 2001 , de nombreuses entreprises ont saisi l'occasion. Si ce mouvement, surtout du côté des banques, s'est d'abord déroulé dans de nombreux cas sous forme d'échanges de partici- 
pations, la tendance s'est accélérée en 2004 et 2005 par la vente de paquets d'actions placées sur le marché. Ainsi, une des participations les plus emblématiques, celle de la Deutsche Bank dans DaimlerChrysler, a été fortement réduite récemment et ne s'élève plus qu'à $6,9 \%$ (après avoir atteint son niveau le plus haut au milieu des années 1970 avec $57,5 \%$, puis être tombée à $12 \%$ en 2000). De plus en plus d'entreprises profitent également de l'occasion pour placer des filiales sur le marché (comme Bayer avec Lanxess).

L'internationalisation de l'actionnariat des entreprises allemandes

\begin{tabular}{|l|rr|rr|}
\hline Entreprise & \multicolumn{3}{|c|}{ Actionnariat dispersé (en \%) } & \multicolumn{3}{|c|}{ Origine étrangère (en \%) } \\
& $\mathbf{2 0 0 1}$ & $\mathbf{2 0 0 5}$ & $\mathbf{2 0 0 1}$ & $\mathbf{2 0 0 5}$ \\
\hline Adidas-Salomon & 100 & 100 & $\left.{ }^{*}\right) 53$ & $\left.{ }^{*}\right) 68$ \\
Allianz & 64 & 100 & 32 & 40,4 \\
BASF & 89,8 & 100 & 35 & 52,4 \\
Commerzbank & 74 & 91 & 37 & 51,5 \\
Continental & 85 & 79,4 & $\left.{ }^{*}\right) 64$ & $\left.{ }^{*}\right) 86,7$ \\
DaimlerChrysler & 81 & 85,9 & nd. & 45 \\
Deutsche Bank & 100 & 93,9 & 53 & 51 \\
Deutsche Börse & 76,5 & 100 & 32 & 65 \\
E.on & 86,8 & 100 & 42,2 & 45,2 \\
Hypo-Vereinsbank & 62,6 & 81,6 & nd & *) 48,12 \\
MAN & 69 & 100 & $*) 19$ & $*) 37$ \\
Schering & 89,4 & 89,4 & 40 & 52 \\
Siemens & 93,5 & 93,5 & 48 & 56,6 \\
Volkswagen & 76,5 & 68,5 & $\left.{ }^{*}\right) 23,3$ & $*$ *) 38,9 \\
\hline Moyenne DAX & $\mathbf{7 2 , 4}$ & $\mathbf{8 1 , 5}$ & $\mathbf{3 5 , 5}$ & $\mathbf{4 3 , 8}$ \\
\hline
\end{tabular}

Source : Handelsblatt du 20 août 2005. *) La part étrangère ne contient que des investisseurs institutionnels et stratégiques (pas de particuliers).

La part des étrangers dans le capital des entreprises allemandes a ainsi nettement augmenté : entre 2001 et 2005, elle s'est accrue de plus d'un quart. Huit entreprises membres du DAX sont aujourd'hui majoritairement contrôlées par des investisseurs étrangers (contre 3 en 2001). L'obligation d'accroître la rentabilité et d'assurer une évolution boursière satisfaisante se renforce. Continental marque ainsi l'exemple : l'entreprise est la plus internationale d'Allemagne (elle est détenue à près de $90 \%$ par des investisseurs étrangers) et a enregistré depuis 2001 la meilleure progression parmi les valeurs du DAX.

\section{L'avenir de Francfort dans la consolidation boursière en Europe}

Après la seconde Guerre mondiale, Francfort est devenu peu à peu le centre financier dominant en Allemagne, sans pourtant entrer dans le cercle des trois grands: New York, Londres et Tokio (la triade). Elle fait partie des places de deuxième catégorie, comme Paris, Amsterdam et Madrid. Mais bien qu'elles soient à un niveau comparable, ces places ne se trouvent pas vraiment dans une situation de concurrence. Plutôt, elles doivent veiller à bien remplir leur rôle face à leur public national (rôle relatif à la culture d'entreprise, aux besoins des $\mathrm{PME}$, etc.) et à protéger leur position face aux concurrents réels, qui se trouvent plus haut et plus bas dans la hiérarchie. En Europe, ce concurrent du haut est Londres pour toutes les places boursières. Dans le cas de Francfort, la concurrence du bas provient d'un côté des bourses régionales allemandes ou des bourses européennes de troisième catégorie, comme Genève ou Luxembourg. Concrètement, s'il s'agit par exemple pour une banque chinoise de s'installer en Europe, elle le fera soit 'seulement à Londres', soit 'à Londres et à Francfort mais elle n'hésitera pas entre Paris ou Francfort. Pour une banque du Maghreb, la question est 'seulement Londres' ou 'seulement Paris' ou éventuellement 'Paris et Londres', mais pas 'seulement Paris' ou 'seulement Francfort'.

Francfort et Paris sont au même niveau 
Les principales bourses européennes en chiffres

\begin{tabular}{|c|c|c|c|c|c|c|}
\hline & $\begin{array}{l}\text { Capitalisation } \\
\text { boursière }^{\text {a) }}\end{array}$ & $\begin{array}{l}\text { Valeur des } \\
\text { titres } \\
\text { échangés }^{\text {a) }}\end{array}$ & $\begin{array}{l}\text { Valeur des } \\
\text { obligations } \\
\text { échangées }\end{array}$ & $\begin{array}{l}\text { Nombre de } \\
\text { sociétés cotées } \\
\text { b) }\end{array}$ & $\begin{array}{c}\text { Coûts de } \\
\text { transaction } \\
\text { et de liquidité }^{c)}\end{array}$ & $\begin{array}{l}\text { Coût de } \\
\text { compensation } \\
\text { (clearing) }^{\text {d) }}\end{array}$ \\
\hline Deutsche Börse & 1995 (+11\%) & 1541 & 442 & 819 (159) & 10,2 & $0,25-0,4$ \\
\hline LSE & 2865 (+17\%) & 5169 & 2793 & 2837 (351) & 37,7 & $0,32-0,9$ \\
\hline Euronext & $2441(+18 \%)$ & 2472 & 232 & $1333(334)$ & 10,8 & $0,3-1,13$ \\
\hline BME & 941 (+29\%) & 1203 & 3553 & e) & 16,8 & nd \\
\hline sWX & $826(+14 \%)$ & 791 & e) & 409 (127) & nd & 0,26 \\
\hline Borsa Italiana & e) & 969 & e) & e) & 19,2 & 0,72 \\
\hline OMX Stockholm & e) & e) & 1541 & e) & nd & nd \\
\hline NYSE & $12708(+13 \%)$ & 11618 & e) & 2293 (459) & 15,5 & nd \\
\hline
\end{tabular}

Sources : Spahn (2003), rapport de Giovanni (2001), www.FIBV.org. a) En milliards de US \$ en 2004. b) Pour 2004, entre parenthèses, le nombre de sociétés étrangères. c) En points de base pour 2002. d) En €., pour une transaction nationale en actions, données pour 2001. e) La bourse ne figure pas parmi les 10 premières places.

Francfort a deux adversaires : Londres et les places régionales
La véritable concurrence internationale se joue vers le bas et vers le haut, mais entre places du même niveau. Pour Francfort, la question est de savoir combien de transactions ou d'emplois elle perd à Londres ou, inversément, réussit à prendre à la capitale britannique. Mais il ne s'agit pas d'atteindre ou même de dépasser Londres. Quant à la concurrence vers le bas, notamment avec les bourses régionales allemandes qui ont su se positionner chacune dans une niche (Berlin dans des valeurs est-européennes, Stuttgart dans les produits dérivés, Munich pour les $\mathrm{PME}$ ), elle est très bénéfique. Francfort à ainsi deux 'rivales' importantes : la bourse de Londres et les places financières régionales, face auxquelles elle a perdu un peu de terrain ces dernières années. Ainsi, les activités de banque d'investissement se pratiquent aujourd'hui essentiellement à Londres ; il ne reste à Francfort que les affaires concernant l'Allemagne. En termes de localisation pour des banques étrangères, Francfort a perdu des parts de marché par rapport à d'autres villes allemandes (Munich, Berlin). Le fait que la Dresdner Bank est maintenant gérée à Munich nourrit cette tendance. Certes, cette perte de poids face à Munich va s'atténuer quand la reprise de la HVB par Unicrédito sera achevée, mais elle entraînera un départ de compétences, d'ingénierie et certainement d'emplois bancaires, affaiblissant l'ensemble de la place allemande.

\section{La consolidation boursière en Europe depuis 1997}

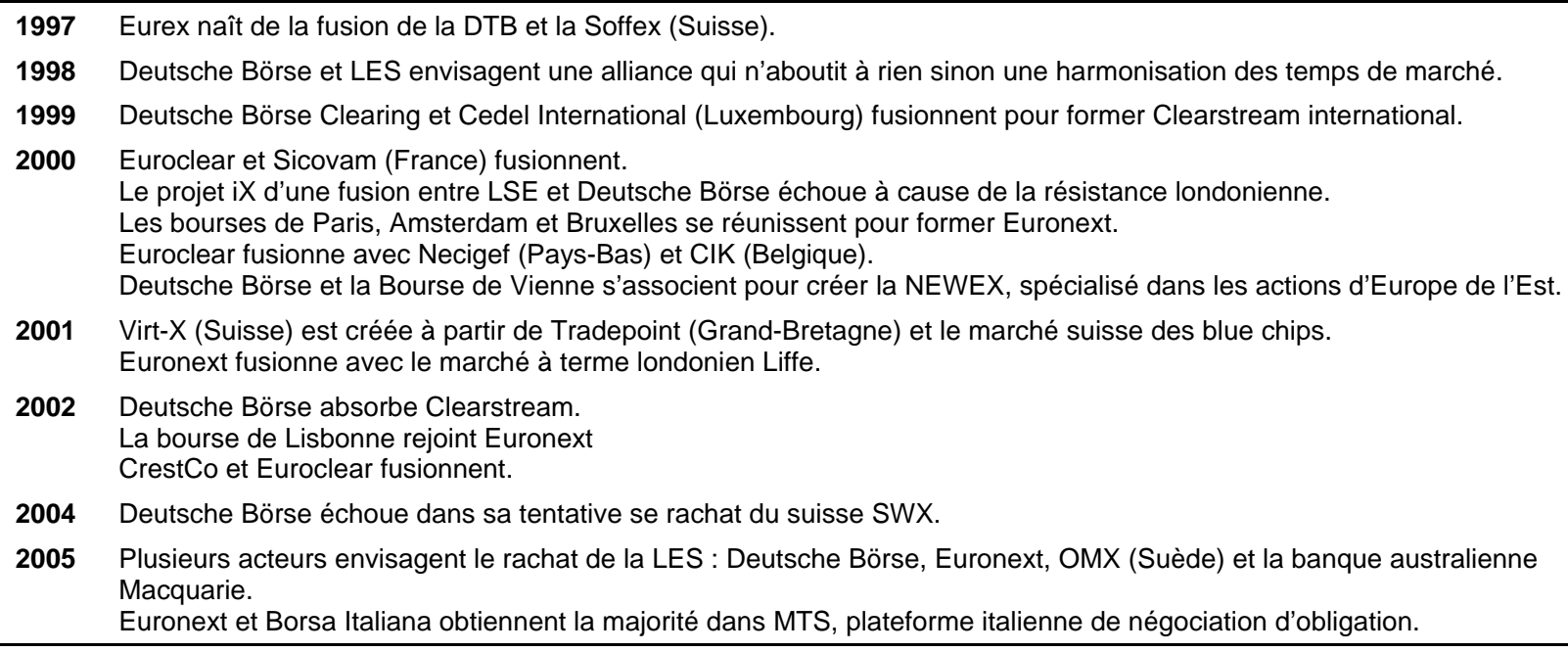

2002 Deutsche Börse absorbe Clearstream.

La bourse de Lisbonne rejoint Euronext

CrestCo et Euroclear fusionnent.

2004 Deutsche Börse échoue dans sa tentative se rachat du suisse SWX.

2005 Plusieurs acteurs envisagent le rachat de la LES : Deutsche Börse, Euronext, OMX (Suède) et la banque australienne Macquarie.

Euronext et Borsa Italiana obtiennent la majorité dans MTS, plateforme italienne de négociation d'obligation.

Sources : Spahn (2003) et actualisation par l'auteur.

L'Europe boursière reste très fragmentée
La Commission européenne déplore depuis longtemps la fragmentation de l'Europe en une quinzaine de bourses. Malgré certains progrès, cette fragmentation persiste. La plupart des marchés sur le continent européen sont verticalement 
intégrés en silos. Ainsi, ils ne laissent aux donneurs d'ordres pas d'autre possibilité que de faire traiter toute la chaîne de réalisation d'une transaction (exécution, compensation, règlement-livraison) par le même opérateur. A cela s'ajoute que chaque marché a ses propres règles comptables et de transparence. En conséquence, les coûts des transactions transfrontalières demeurent nettement plus élevés que ceux des transactions nationales. La différence avec les USA où coexistent seulement deux systèmes de paiement et trois systèmes de règlement-livraison est frappante. On n'y compte que deux bourses dominantes qui, de surcroît, agissent en segments clairement séparés : le NYSE pour les valeurs traditionnelles et le Nasdaq pour les valeurs technologiques. A ces problèmes s'ajoute que, sur les places européennes, un nombre très restreint de titres représente une part importante (parfois plus de la moitié) de l'ensemble des transactions. L'existence de ces champions nationaux pose un problème sérieux de liquidité et d'accès aux marchés, notamment pour la cotation des PME.

Les champions nationaux dans les transactions d'ensemble en Europe

\begin{tabular}{|c|c|c|c|c|c|}
\hline Bourse & Société & $\begin{array}{c}\% \text { du volume de } \\
\text { transactions en } 2003\end{array}$ & Bourse & Société & $\begin{array}{c}\% \text { du volume de } \\
\text { transactions en } 2003\end{array}$ \\
\hline Londres & $\begin{array}{l}\text { Vodafone } \\
\text { HSBC }\end{array}$ & $\begin{array}{l}5,9 \\
5,8\end{array}$ & Milan & $\begin{array}{l}\text { STM } \\
\text { Eni }\end{array}$ & $\begin{array}{r}19,21 \\
9,5\end{array}$ \\
\hline Francfort & $\begin{array}{c}\text { Allianz } \\
\text { Münch. Rück }\end{array}$ & $\begin{array}{l}7,8 \\
7,8\end{array}$ & Madrid & $\begin{array}{c}\mathrm{SCH} \\
\text { Telefonica }\end{array}$ & $\begin{array}{l}21,3 \\
19,6\end{array}$ \\
\hline Paris & $\begin{array}{c}\text { Total } \\
\text { France Télécom }\end{array}$ & $\begin{array}{l}9,7 \\
5,2\end{array}$ & Zurich & $\begin{array}{c}\text { Logitec } \\
\text { Converium }\end{array}$ & $\begin{array}{r}11,4 \\
7,8\end{array}$ \\
\hline
\end{tabular}

Source : Fédération des bourses de valeurs européennes (www.fese.org).

Si l'essor de la consolidation boursière européenne reste tributaire de l'harmonisation de la réglementation, elle se heurte aussi au prestige national. Une consolidation rapide est pour l'instant hors de portée. Une voie plus souple, celle des coopérations entre bourses, promet plus de succès. Combinées à une harmonisation de la réglementation, elles évitent d'un côté de payer des prix d'achat trop élevés et préservent de l'autre un certain niveau de concurrence, évitant ainsi des situations de monopole. D'importantes économies de coûts pourraient par ailleurs être réalisées par la centralisation des domaines de la compensation et du paiement-livraison. Enfin, le processus de consolidation ne doit pas seulement se faire entre les opérateurs de marché. Ces derniers sont aujourd'hui essentiellement des entreprises de services en TIC. La DBAG vend son expertise technologique et ses produits à d'autres opérateurs. On a ainsi assisté à une consolidation de l'infrastructure boursière ces dernières années : Vienne, Dublin, Helsinki et la bourse de l'électricité EEX tournent avec de la technologie DBAG. Non seulement la complexité de l'infrastructure boursière se réduit ainsi, elle diminue également les coûts, car la technologie boursière souffre par nature de coûts fixes élevés. La technologie devient ainsi le véritable facteur de compétitivité. Celle-ci se génère de moins en moins au plan du rendement et de la vitesse (les systèmes ne se distinguent plus que par quelques détails), mais à un autre niveau : celui de la capacité à fournir l'information financière à toutes les parties concernées par la transaction à tout moment et en temps réel (straight through processing). Les investisseurs exigent aujourd'hui de pouvoir suivre leurs transactions tout au long de la chaîne de réalisation avec la possibilité d'y intervenir à tout moment. La DBAG est bien positionnée dans ce domaine.

LE CAS DE LA DeUTSChe BöRSE AG traduit parfaitement l'actuelle mutation de la Deutschland $A G$ : elle s'internationalise, accroît sa transparence et donne plus de pouvoirs aux actionnaires. Pour la place financière de Francfort, l'échec du projet n'est finalement pas une mauvaise nouvelle. Elle y aurait perdu l'un de ses éléments-phares. Mais la place de Francfort n'est pas pour autant dans une
L'Europe boursière : plus de réseaux et plus de consolidation technologique 
situation très confortable. Non seulement elle est attaquée de deux côtés, Londres et les places financières régionales, mais elle est également confrontée à d'autres problèmes qui se situent plus en interne. Ainsi, les politiques menées par les différents gouvernements se sont trop exclusivement concentrées sur l'aspect boursier et marché des capitaux. Une place financière est cependant bien plus que cela: elle inclut également les banques. Ainsi, les différentes lois visant la promotion des marchés financiers (Finanzmarktförderungsgesetze) adoptées ces dernières années ont certes été bénéfiques pour la culture boursière et d'investissement, mais elles ont aussi été des lois d'affaiblissement pour les banques. Rappelons qu'il existe une relation étroite entre les éléments de chaque système financier. Dans le système économique allemand, les banques jouent traditionnellement un rôle central, à travers le canal du crédit bancaire. Malgré l'ouverture au modèle anglo-saxon, cette préférence pour le crédit bancaire ne s'est pas vraiment réduite. La complémentarité entre les éléments du système financier est une condition essentielle pour sa santé.

L'affaiblissement des banques en interne et la prise de contrôle par des concurrents étrangers représentent probablement le plus grand risque pour la place financière de Francfort. La politique devrait être consciente de ce risque. Enfin, la place de Francfort sera également affectée par une autre tendance, cette fois-ci commune à toutes les places. II s'agit de l'internalisation des ordres de bourse, pratique de marché qui consiste pour un intermédiaire financier, souvent les banques, à exécuter directement les ordres de ses clients en interne sans les exposer au marché réglementé. Dans le cadre de son programme d'activation des marchés financiers, la Commission européenne autorise ces pratiques à partir de 2007 - une raison de plus pour que Francfort trouve encore avant cette date une taille critique suffisante pour ne pas être marginalisée en tant que place boursière.

\section{Indications bibliographiques :}

GABEL M., "La banque-industrie et les industries bancaires », Regards sur l'économie allemande, $\mathrm{n}^{\circ} 45$, mars 2000

GABEL M., " La NEWEX de Vienne. Nouvel acteur sur les marchés d'actions de l'Est ? ", Courrier des pays de l'Est, août 2001

Holzhausen A., «Finanzplatz Deutschland », Working paper n²8, Allianz Group, Dresdner Bank, 2004

SChmidt R. H., Tyrell M. et HACKethal A., "Corporate Governance in Germany : Transition to a Modern Capital Market-Based Financial System ? Views and Comments ", Journal of Institutional and Theoretical Economics (JITE), vol. 159, 2003

SCHMIDT R. H. et GROTE M. H., "Was ist und was braucht ein bedeutender Finanzplatz ? ", Working paper series, Finance and Accounting, n`150, Goethe Universität , Francfort/Main, avril 2005

Spahn B. et Van Den Busch U., Position und Entwicklungsperspektiven des Finanzplatzes Frankfurt, FEH-Report n645, Wiesbaden, 2002

Vitols S., "Changes in Germany's Bank-Based Financial System: A varieties of Capitalism perspective », Discussion paper SP II, Wissenschaftszentrum Berlin, n³, 2004. 